\title{
Forum
}

\section{Marine Radar: A Selective Review of R\&D and a Way Ahead}

\author{
P. D. L. Williams
}

Commercial marine radar has to perform as well as many military systems but for a fraction of the price. Remote sensing radars are as sophisticated as military ones and both have engendered $R \& D$ (research and development) which can be used at sea, especially for the detection of small surface targets in clutter. This $R \& D$ can also indicate a way ahead for civil sets into the 2 Ist century.

1. INTRODUCTION. Merril Skolnik once commented to the author that, while there were plenty of textbooks and a mass of literature each month devoted to radar for use in aircraft, in space and for military purposes, the civil marine people often had to refer back to Wylie's 1968 book $^{1}$ and Joe Croney's chapter in his (Skolnik's) own 1970 handbook. ${ }^{2}$ There are many papers relating to marine radar but their application is not always obvious and, whilst a new marine radar textbook seems desirable, it could not hope to embrace all the possible contributions required for marine sets.

The author has published some 25 papers on marine radar, partly to report original work and partly to bridge the gap between the enormous literature now available and the requirements of marine radar designers and users. So this already shortened paper is aimed at persuading young marine radar design engineers to examine both old and new techniques to improve their products for the next century.

2. POST-1970 IMPROVEMENTS. Since Croney's review of marine radar in $1970^{2}$ the introduction of solid state devices and the widespread use of integrated circuits has reduced power consumption and improved reliability, thus enabling many digital circuits to be used to reduce drift and improve setting up procedures. The analogue, real time, PPI is now almost a museum piece, replaced by the digital scan converter (DSC) often driving a colour display.

3. R\&D FOR SMALL TARGET DETECTION

3.1. The problem of small target detection provides a focus for a range of techniques. The ice growler and the waterlogged container floating with little freeboard ${ }^{3}$ are typical hazards. The five main reasons why detection of these difficult targets is both hard to estimate and hard to carry out are:

(a) Too low a signal to thermal noise ratio for the given scenario of target, radar and range in free space as calculated from the basic radar equation.

(b) The limited radar horizon for a given target and radar scanner height, and the dependence of the transition range on the operating wavelength.

(c) Too low a target to clutter ratio for a given foul weather scenario together with the use of incorrect or optimistic models for both area and volume clutter and targets.

(d) The rapid, frequent and serious deviations from propagation theory particularly over water and often as a function of azimuth. 
(e) Actual blockage by waves and/or swell occurring between the radar scanner and the target.

3.2. Short comments on the above lettered subsections are as follows:

(a) This problem will only occur with very low performance sets but is a cause of worry to sailors who see a floating object visually but not on the radar. The solution is to use realistic median values for marine targets as suggested by Williams, ${ }^{3}$ and to include the causes of the many radar signal losses in design calculations.

(b) This can be a limitation in calmer seas when the scanner is too low, particularly with $10 \mathrm{~cm}$ radars.

(c) Later in this paper, solutions to the problem of too low a target to clutter ratio will be discussed. A review of techniques up to 1970 is given in the chapters on sea echo and weather (clutter) effects on radar as well as in the chapter on civil marine radar in Skolnik. ${ }^{2}$ Williams ${ }^{4}$ gave a review of growler detection in 1979 after joint studies in Canada and, since then, ice and growler detection and recognition have been featured at annual radar conferences (see also Lewis et al. on ice detection in 1987). ${ }^{5}$

(d) Variations in radar cross-sections (RCs) are often difficult to distinguish from changes in path length attenuation due to precipitation, multipath, anomalous propagation and target blockage. Many trials show the advantages of diversity in operation-for example, by using twin radars at different heights, two carrier frequencies, multiple polarizations etc..$^{3,6}$

(e) Target fade due to wave blockage is less well researched than fade due to sea clutter characteristics, as will be discussed. It does not feature in the standard Swerling models designed for aircraft targets.

4. REView OF R\&D APplications and techniques. Some of the R\&D applications and techniques are available for the following purposes:

(i) Receiver gain or threshold control.

(ii) Choice of radar parameters.

(iii) Recognition of different characteristics of target and clutter.

(iv) Use of multiple radar inputs/diversity.

4.1. Receiver gain control. Until the 1960s only simple manual gain and swept gain controls were provided at sea, though instantaneous automatic gain control (IAGC) had been in military radars 20 years before. Then Croney introduced constant false alarm rate (CFAR) techniques ${ }^{7}$ to marine radar as a prelude to his fast scanning research. ${ }^{2}$

For noise like clutter, the log receiver and hi-pass filter provide good CFaR gain or threshold control in precipitation but, for modest to high spatial resolution. radars, particularly using horizontal polarization for both transmission and reception (HH) in sea clutter, 'spikes' occur which look and behave like targets. These have remained a problem ever since. Reports of such spikes began in $1957^{8}$ but were not addressed in marine radar till much later.

4. 2. Choice of radar parameters. Initial comments on five major reasons why detection of small surface targets is hard to estimate and hard to achieve have already been made. A more detailed discussion is now attempted.

Free space clutter-free performance. First it is assumed that sufficient power, antenna gain and aperture, and receiver noise factor are available to detect the smallest $\operatorname{target}^{3}$ at the required range, with margins for extra attenuation due to precipitation and surface moisture, though there is always some uncertainty since low layers of moist air may cause ducting.

Additional propagation attenuation. Sometimes the curved Earth and transition range are the dominant effects causing performance loss and, together with antenna size, these 
factors favour $3 \mathrm{~cm}$ wavelength marine radar. However, sea moisture can both increase or decrease path loss.

We go on to examine operating frequency and bandwidth, spatial and volume resolution, and sampling methods. Finally, platform motion has to be considered together with signal processing and target integration methods to produce satisfactory track history.

Wavelength. In 1975, a comprehensive analysis of choice of wavelength was made by comparing the performance of two radars equal in all respects except antenna size; that is, with the same horizontal and vertical beamwidth. This was followed by a series of trials for targets within the transition range of both radars, and then in sea clutter and rain clutter for a wide range of maritime targets including coastal features at long ranges. ${ }^{9}$ Both theory and trials showed the benefits and drawbacks of using either wavelength and also many examples of diversity operation. It must be recognized that the longer $10 \mathrm{~cm}$ wavelength radar generally requires an antenna three times as big as the $3 \mathrm{~cm}$ one. However, it is possible for sets with equal aperture size to offer different features such as better angular resolution on the $3 \mathrm{~cm}$ band; for example, the Racal Decca $2459 \mathrm{~F} /$ I. $10 .^{10}$ Horizontal beam width and range resolution determine the spatial resolution but, for twenty years, the appearance of clutter spikes has spoilt the improvements expected through the use of narrow beams and short pulses, with or without compression, at sea although this has been less of a problem in the air.

Angular resolution. Apart from target resolution, simple theory recommends the use of narrow beams but, with a beamwidth of less than 2 degrees, the appearance of sea clutter spikes has been a problem for many years. At sea, a ship's yaw reduces the chance of good target overlap on successive scans and, consequently, integration and track history is spoilt. Even with a compass-stabilized display, roll and pitch can corrupt angular data due to the canted trunnion effect. A gimbal mounting can reduce this effect, but a planar array with limited electronic scanning is even more efficient since it is able to put a narrow horizontal and vertical beam on the sea surface when controlled from a vertical sensor such as a ring laser gyro as well as an azimuth input from a transmitting compass. The DSC together with two-dimensional electronic scanning enables the display to be always correct and even allows a non-progressive scanning motion to be accommodated as a ship falls back off a wave; that is, the look-back is truly mapped. Single axis electronic scanning allows beam steering in the 'vertical' axis only and a solid state transmitter with a vertically dispersive array can steer the beam by changing carrier frequency so that the antenna is entirely passive with no need for active phase shifters, slip rings etc. ${ }^{11}$ The narrow beam gives two more benefits. First, the smaller vertical beam width with bigger vertical aperture provides more system gain and aperture, which allows the employment of all solid state pulse transmitters without pulse compression as well as even lower power FM/Cw sets than the Kelvin Hughes Scout which is based on the Philips research by Stove et al. ${ }^{12}$ Secondly, the rain clutter problem is reduced by a ratio of about 20 degrees to 2 degrees or $10 \mathrm{db}$.

Polarization. Croney and Schneider provide good reviews of this topic up to $1976,{ }^{6}$ but workers in the remote sensing field have taken the work much further for target recognition. ${ }^{13,14,15}$ Nevertheless, proven advances in automatic rain rejection, coupled with up to $30 \mathrm{~dB}$ recovery of fading targets by the use of twin channel receivers to receive both the co-polar and cross-polar signals, have still not been used at sea. Such techniques also allow transponder operation, the absence of which limited the use of the old Sperry Sea Track radar. ${ }^{2}$

Range resolution. Again, sample theory favours short pulse lengths for good range resolution. This principle is borne out in aircraft trials with moderate angular resolution 
(and steeper grazing angles) but it tends to produce unwanted spikes with narrow beams using HH. Olin ${ }^{16}$ has shown sea clutter spikes correlating with optical pictures of patches of excessive wave activity in the open sea prior to them breaking up and dispersing in foam. In addition, shadowing effects and Bragg scattering ${ }^{17}$ are now all thought to contribute to spikes.

Olin $^{18}$ later reported measurements of both amplitude and temporal statistics of spikes as a function of sea state. A spike was defined as having an rcs greater than i sq metre, showing how serious a problem they present. The paper also gave details of spike width and spacing as well as their fast fluctuation rates. Practical problems of rapid relative motion between two fast closing boats also spoil scan-to-scan correlation as a method of improving target recognition against spike clutter.

4.3. Recognition of different characteristics of targets and clutter. The efforts spent in setting receiver thresholds arose because straight selection by amplitude was all that was available for many years. Filtering in the doppler frequency domain was first adopted in early MTI sets and later in pulse doppler sets, which now operate with adaptive clutter notches, variable in both notch frequency and width, to work from moving platforms and handle different patches of clutter. These are very successful when clutter and targets have a significant radial speed differential but this is not present in crossing targets, apart from helicopters. Attention was therefore given to different amplitude or fade rates of targets and sea clutter by raising scanning rates to perceive clutter twinkle. ${ }^{2}$ However, this technique failed in higher sea states, possibly due to target modulation from multipath and rapid obscuration.

Workers in the remote sensing field are now using both multi-frequency and multipolarization radars in airborne experiments prior to similar equipment being used in space, but they are mainly interested in target classification. ${ }^{13},{ }^{4,15}$ However, they cannot easily take long looks at scenes and targets during each pass to examine fade rates as Croney and others have done.

Once a true target at sea has been detected and had an ARPA tracker put on it, it is often tracked through heavy fades on its projected course and speed, but this is no help for detecting unknown targets unless there are dozens of tracker circuits set up waiting to capture appropriate targets that fit their parameters! This is similar to the production of aborted tracks in air traffic control.

4.4. Use of multi-radar inputs/diversity. Diversity operation can look for target detection in any one of two or more channels. This results in a diversity gain, although perhaps with a higher false alarm rate unless proper CFAR techniques are used prior to channel/signal combination. Otherwise, diversity can be used to confirm target detection by means of a logical " and" operation. This results in lower false alarm rates and reduced sensitivity but is effective when the drop in false alarm rate is greater than the reduced target detection. Indeed, the video combination circuits in the Racal-Decca $2459 \mathrm{~F} / \mathrm{I}$ allowed either mode to be used, but it was never fully evaluated. A particular case of limited frequency diversity is the pulse to pulse carrier agility used to force sea clutter decorrelation ${ }^{19}$ as tested and verified by Croney ${ }^{2}$ and many others since.

5. SYSTEMS FOR TODAY AND TOMORROW. The choice of radar parameters and techniques is, as in most designs, a technical compromise, even apart from cost and size restraints. However, the DSC and the PC/VDU have made dedicated display manufacture no longer necessary since the real-time radar video stops at the DSC. In the same way, solid-state transmitters and $\mathrm{FM} / \mathrm{cW}$ will make a radar head more like a communications transceiver, with a non-real-time video circuit even before the DSC stage. This means that by $2000 \mathrm{AD}$, one equipment will be able to operate in various modes appropriate to target detection and tracking in various forms of clutter with different $\mathrm{T} x$ waveforms 
and spatial resolution perhaps changing many times during a scan. This may occur even before full 360 degree scanning is brought in together with sophisticated Tx energy management techniques. ${ }^{20}$ Indeed, in Canada, Haykin ${ }^{21}$ is using his IPIX equipment to develop a system approach to identify a way ahead.

6. THE FUTURE. As to the future, a number of predictions are made.

6.1. Fully solid-state radars and displays will be in place by the year 2000 and will allow flexible transmitter frequency control at first for vertical electronic scanning, for agility to improve clutter decorrelation and for $\mathrm{FM} / \mathrm{CW}$ operation.

6.2. More use will be made of small solid-state gyros for compass-stabilized displays.

6.3. Partial electronic scanning will produce narrow vertical beam antennas with all ship's angular motion removed.

6.4. Tracking circuits from rapid response compasses/gyros will enable course up displays to be far better stabilized by the removal of canted trunnion error, and also to indicate true course made good with a separate indication of ship's head at any time. ${ }^{22}$

6.5. The cathode ray tube (CRT) will be a museum piece with no supply above $100 \mathrm{~V}$.

6.6. Doppler Beam Sharpening may be used to improve angular resolution with modest aperture antennas. ${ }^{23,24}$

6.7. Inverse SAR (ISAR) techniques will be used to aid large ship recognition in a spotlight mode and this, together with many other military/remote sensing techniques will be introduced via harbour and coastal radars. ${ }^{24}$

6.8. Artificial intelligence using neural nets will be in use. ${ }^{24}$

\section{REFERENCES}

1 Wylie, F. J. (ed.) (1952). The Use of Radar at Sea. Hollis and Carter, revised 1968.

2 Skolnik, M. I. (ed.) (1970). Radar Handbook. McGraw-Hill Book Co., New York, London.

9 Williams, P. D. L., Cramp, H. D. and Curtis, K. (1978). Experimental study of the radar cross-section of maritime targets. Electronic Circuit and Systems, 2, July.

4 Williams, P. D. L. (1979). The detection of ice at sea by radar. The Radio and Electronic Engineer, 49, no. 6, June.

${ }^{3}$ Lewis, E. O. et al. (1 987). Detection and Classification of Ice. Research Studies Press Ltd., John Wiley \& Sons, New York, Chichester, UK.

6 Schneider, A. B. and Williams, P. D. L. (1976). Circular polarization in radars. An assessment of rain clutter reduction and likely loss of target performance. The Radio and Electronic Engineer, 47, I1-29, Jan.-Feb.

3 Croney, J. (1956). Clutter on radar displays: reduction by the use of logarithmic receivers. Wireless Engineer, 33, 83-96, April.

8 MacDonald, F. C. (1 957). Characteristics of radar sea clutter : persistent target-like echoes in sea clutter. USN NRL 4902, March.

${ }^{9}$ Williams, P. D. L. (1975). Limitations of radar techniques for the detection of small surface targets in clutter. The Radio and Electronic Engineer, 45, no. 8.

Po Williams, P. D. L. (1982). Results from a new dual band dual purpose search radar for sea surface and aircraft search. Radar 82 IEE Conf. Pub. no. 216 , pp. 24-29, Oct.

$"$ Rudge, A. W., Milne, K., Olver, A. D. and Knight, P. (1983). The Handbook of Antenna Design Vol. 2. Peter Peregrinus, IEE.

12 Barrett M., Reits, B. J. and Stove, A. G. (1987). An X-band FMCw navigation radar. Radar 87 IEE Conf. Pub. no. 28, Oct., and later, the Kelvin Hughes brochure for their dual mode 'Scout Radar' 1993.

13 Giulo, D. (1986). Polarization diversity in radars. Proc. IEEE Vol. 74, no. 2, PP. 245-269 [1 25 refs].

${ }_{14}$ Zebker, H. A. et al. (1991). Calibrated imaging radar polarimetry: technique, examples, and applications. IEEE Transactions on Geoscience and Remote Sensing, Vol. 29, no. 6, Nov.

15 Freeman, A. (1992). SAR calibration: an overview. IEEE Transactions on Geoscience and Remote Sensing, Vol. 29, no. 6, Nov. [1 27 refs]. 

Oct.

16 Olin, I. (1977). Some recent observations of sea spikes. Radar 77 IEE Conf. Pub. no. I 55,

17 Trizna, D. B. (1985). Open ocean radar sea scatter measurements. Proc. IEEE 1985 International Radar Conference. Arlington, USA. IEEE Cat. no. ${ }_{5} \mathrm{CH}_{20} 076-8$.

18 Olin, I. (1982). Amplitude and temporal statistics of sea spike clutter. Radar 82. IEE Conf. Pub. no. 216 , Oct.

19 Nathansen, F. (1969). Radar Design Principles. McGraw-Hill Book Co., New York.

20 Billam, E. R. (1992). Parameter optimization in phased array radar. Radar 92. IEE Conf. Pub. no. 365 , Oct.

21 Haykin, S. (1991). Adaptive multidimensional signal processing for ocean surveillance using surface based radar. Conference Record of the 25 th Asilomer Conference on Signals, Systems and Computers. IEEE Cat. no. $91 \mathrm{CH}_{3} 1$ 2-o, Pacific Grove, CA, USA.

${ }_{22}$ Williams, P. D. L. (1992). Pat. App. no. 9,220,982.4.

23 Morchin, W. (1993). Radar Engineer's Sourcebook. Artech House, Boston, London.

24 Radar 92. IEE Conf. Whole sessions on ISAR/DBS/AI/Neural nets.

I. Radar. 2. Marine radar.

\section{Erratum}

There was an error in Mr Richard Trim's paper, 'Some causes of problems in the observation of standard racon marine beacons when observed by means of standard marine navigation radars', which was published in the May 1993 issue of the Journal of Navigation. Section 3, paragraph 4 of page 276 should read:

'A third and very important cause of radar received-signal differentiation arises if a widely used form of automatic anti-sea-clutter processing is employed, since part of this processing is to differentiate the radar-received video so as to remove the d.c. term in the sea clutter echoes as part of the Constant False Alarm Rate (CFAR) processing. When such automatic sea clutter supression facilities are in operation, the gain level applied to the radar receiver video amplifier has an adaptive signal superimposed upon it which, while slow acting, generally follows the shape of the clutter returns on the received signal video, while being largely unaffected by the wanted echo returns such as those from ships, navigation marks, coastlines, etc. This effect may be reduced in the case of the very latest radar designs'. 